\title{
Outcomes of COVID-19 in Patients With a History of Cancer and Comorbid Cardiovascular Disease
}

\author{
Sarju Ganatra, MD ${ }^{1, *}$; Sourbha S. Dani, MD ${ }^{1, *}$; Robert Redd, MS²; Kimberly Rieger-Christ, PhD; Rushin Patel, MD ${ }^{1}$ \\ Rohan Parikh, MD1; Aarti Asnani, MD4; Vigyan Bang, MD; Katherine Shreyder, MD1; Simarjeet S. Brar, MD5;

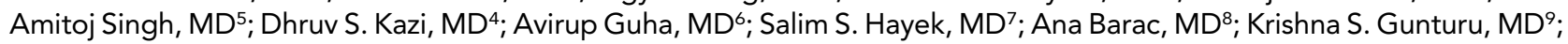 \\ Corrine Zarwan, MD ; Anne C. Mosenthal, MD ${ }^{10}$; Shakeeb A. Yunus, MD ${ }^{11}$; Amudha Kumar, MD ; Jaymin M. Patel, MD ${ }^{12}$; \\ Richard D. Patten, MD'; David M. Venesy, MD'; Sachin P. Shah, MD¹; Frederic S. Resnic, MD; \\ Anju Nohria, MD ${ }^{13, *}$; and Suzanne J. Baron, MD ${ }^{1, *}$
}

\begin{abstract}
Background: Cancer and cardiovascular disease (CVD) are independently associated with adverse outcomes in patients with COVID-19. However, outcomes in patients with COVID-19 with both cancer and comorbid CVD are unknown. Methods: This retrospective study included 2,476 patients who tested positive for SARS-CoV-2 at 4 Massachusetts hospitals between March 11 and May 21, 2020. Patients were stratified by a history of either cancer $(n=195)$ or CVD $(n=414)$ and subsequently by the presence of both cancer and CVD $(n=82)$. We compared outcomes between patients with and without cancer and patients with both cancer and CVD compared with patients with either condition alone. The primary endpoint was COVID-19-associated severe disease, defined as a composite of the need for mechanical ventilation, shock, or death. Secondary endpoints included death, shock, need for mechanical ventilation, need for supplemental oxygen, arrhythmia, venous thromboembolism, encephalopathy, abnormal troponin level, and length of stay. Results: Multivariable analysis identified cancer as an independent predictor of COVID-19-associated severe disease among all infected patients. Patients with cancer were more likely to develop COVID-19-associated severe disease than were those without cancer (hazard ratio [HR], 2.02; 95\% Cl, 1.53-2.68; $P<.001)$. Furthermore, patients with both cancer and CVD had a
\end{abstract}

\footnotetext{
${ }^{1}$ Division of Cardiovascular Medicine, Department of Medicine, Lahey Hospital and Medical Center, Burlington, Massachusetts; ${ }^{2}$ Department of Data Science, Dana Farber Cancer Institute, Boston, Massachusetts; ${ }^{3}$ Department of Translational and Cancer Research, Lahey Hospital and Medical Center, Burlington, Massachusetts; ${ }^{4}$ Division of Cardiovascular Medicine, Department of Medicine, Beth Israel Deaconess Medical Center, Boston, Massachusetts; ${ }^{5}$ Department of Medicine, Lahey Hospital and Medical Center, Burlington, Massachusetts; ${ }^{6}$ Harrington Heart and Vascular Institute, Case Western Reserve University School of Medicine, Cleveland, Ohio; ${ }^{7}$ Division of Cardiovascular Medicine, University of Michigan, Ann Arbor, Michigan; ${ }^{8}$ Department of Cardiology, MedStar Washington Hospital Center, MedStar Heart and Vascular Institute, Washington, DC; ${ }^{9}$ Division of Hematology-Oncology, Department of Medicine, Lahey Hospital and Medical Center, Burlington, Massachusetts;

${ }^{10}$ Department of Academic Affairs, Lahey Hospital and Medical Center, Tufts University School of Medicine, Burlington, Massachusetts; ${ }^{11}$ Division of Hematology-Oncology, Beverly Hospital, Beverly, Massachusetts; ${ }^{12}$ Division of Hematology-Oncology, Department of Medicine, Beth Israel Deaconess Medical Center, Boston, Massachusetts; and ${ }^{13}$ Division of Cardiovascular Medicine,

Department of Medicine, Brigham and Women's Hospital, Boston, Massachusetts.

*These authors have contributed equally to this study.
}

higher likelihood of COVID-19-associated severe disease compared with those with either cancer $(\mathrm{HR}, 1.86 ; 95 \% \mathrm{Cl}, 1.11-3.10 ; \mathrm{P}=.02)$ or CVD (HR, 1.79; 95\% Cl, 1.21-2.66; $P=.004)$ alone. Patients died more frequently if they had both cancer and CVD compared with either cancer $(35 \%$ vs $17 \% ; P=.004)$ or CVD (35\% vs $21 \% ; P=.009)$ alone. Arrhythmias and encephalopathy were also more frequent in patients with both cancer and CVD compared with those with cancer alone. Conclusions: Patients with a history of both cancer and CVD are at significantly higher risk of experiencing COVID-19-associated adverse outcomes. Aggressive public health measures are needed to mitigate the risks of COVID-19 infection in this vulnerable patient population.

J Natl Compr Canc Netw, doi: 10.6004/jnccn.2020.7658 Published online November 3, 2020

\section{Background}

COVID-19 caused by the SARS-CoV-2 virus has affected millions of people globally. Early reports have suggested that patients with advanced age and other comorbid conditions, particularly cancer and cardiovascular disease (CVD), face the highest risk of mortality. ${ }^{1-8}$ Although both cancer and CVD have been independently associated with higher COVID-19-associated adverse outcomes, outcomes for patients with COVID-19 afflicted with a dual diagnosis of cancer and CVD are not well understood. To address these gaps in knowledge, we used data from the Lahey Health System to assess COVID-19-associated outcomes in patients with a history of cancer and to evaluate whether patients with both cancer and comorbid CVD are at greater risk than those with either condition alone.

\section{Methods}

\section{Study Oversight}

This study was approved by the Lahey Hospital and Medical Center Institutional Review Board. Written

See JNCCN.org for supplemental online content. 
informed consent was waived. Data were analyzed and interpreted by the authors. All authors reviewed the manuscript and affirm the accuracy and completeness of the data.

\section{Study Population and Design}

This retrospective study included all patients tested for SARS-CoV-2 at 4 medical centers in Massachusetts (Lahey Hospital and Medical Center, Lahey Medical Center, Winchester Hospital, and Beverly Hospital) between March 11 and May 21, 2020. Patients were determined to have a history of cancer if they had any active or prior solid or hematologic malignancy, regardless of the need for therapy. Noninvasive cancers, including nonmelanomatous skin cancer, in-situ carcinoma, or precursor hematologic neoplasms, were not included in the definition of cancer. Patients were determined to have CVD if they had a history of coronary artery disease, cardiomyopathy, congestive heart failure, moderate to severe valvular heart disease, or ischemic stroke.

\section{Data Acquisition}

Patient demographics, comorbidities (including history of cancer, cardiovascular risk factors, preexisting CVD, chronic kidney disease [CKD], and chronic obstructive pulmonary disease [COPD]), details about cancer and cardiovascular treatment, clinical symptoms or signs, laboratory and imaging findings, and clinical events for hospitalized patients were extracted from the electronic medical record (EMR). For both hospitalized and ambulatory patients, any available records from the day of SARS-CoV-2 testing through May 21, 2020, were examined. Some parameters were collected by leveraging EMR informatics. For these parameters, $20 \%$ of the sample was manually cross-checked. In cases of discrepancy between manually and EMR-generated data, 2 independent investigators (S.G., S.S.D.) verified the information. Follow-up of patients treated in the ambulatory setting and postdischarge was conducted at the discretion of the treating physician.

\section{Study Endpoints}

The co-primary endpoints of this study were to assess COVID-19-associated severe disease (composite of need for mechanical ventilation, shock, or death) in patients with cancer compared with those without cancer, ${ }^{1}$ and in patients with both cancer and CVD compared with those to either diagnosis alone. ${ }^{2}$ Secondary endpoints included death, shock, need for mechanical ventilation, need for supplemental oxygen, arrhythmia, venous thromboembolism, encephalopathy, abnormal troponin level, and length of stay in patients with cancer compared with those without cancer ${ }^{1}$ and in patients with both cancer and CVD compared with those to either diagnosis alone. ${ }^{2}$ The definitions of individual endpoints are provided in supplemental eAppendix 1 (available with this article at JNCCN.org).

\section{Statistical Analysis}

Continuous variables are presented as median (range) and compared using the Wilcoxon rank-sum test. Categorical variables are presented as counts (\%) and compared using chi-square or Fisher exact tests. Multivariable logistic regression analysis was performed to identify predictors of COVID-19-associated severe disease in all infected patients. Covariates included in the model were age, sex, history of cancer, CVD, hypertension, diabetes mellitus, tobacco use, CKD, COPD, use of angiotensin-converting enzyme inhibitors or angiotensin receptor blockers, and use of $\beta$-blockers. Variable selection was performed in 2 steps: (1) a random forest was fit with all covariates to evaluate variable importance and predictive variables (determined by first-order depth from root to parent node) of severe disease were selected for further evaluation, and (2) a stepwise, forward/backward model selection was performed using the Akaike information criterion to find a parsimonious set of predictors for the final model. Multivariable logistic models were reported with odds ratios and $95 \%$ confidence intervals. Variance inflation factors (VIFs) were computed for the final model to evaluate multicollinearity. VIFs $>5$ were used to identify highly correlated variables; however, none of the variables included in the model had a VIF $>1.5$, suggesting low multicollinearity.

Time to severe disease was defined as the time from the positive SARS-CoV-2 test to the first occurrence of either mechanical ventilation, shock, or death. The impact of a history of cancer on COVID-19-associated severe-disease-free survival was described using KaplanMeier analyses and was compared with patients without cancer using log-rank tests. Similarly, the impact of cancer and CVD and the combination of cancer and CVD on severe-disease-free survival was described using Kaplan-Meier analysis and compared using logrank tests. Patients without events were censored at the last date of follow-up. Univariate Cox regressions were performed for the time-to-severe disease endpoint, and hazard ratios (HRs), 95\% confidence intervals, and Wald $P$ values were reported; variables were evaluated for and did not violate the proportional hazards assumption. Other adverse effects were compared between groups using the chi-square or Fisher exact tests. A $P$ value $<.05$ was considered statistically significant. Analyses were performed using $\mathrm{R}$ version 4.0.0 (R Foundation for Statistical Computing) and packages "randomForestSRC" (version 2.9.3) and "survival" (version 3.2-3). 


\section{Results}

\section{Patient Population}

Among 21,217 patients tested between March 11 and May 21, 2020, 2,476 tested positive for SARS-CoV-2. Among positive patients, $195(7.9 \%)$ had a history of cancer, 414 (16.7\%) had a history of CVD, and 82 (3.3\%) had both cancer and CVD (Figure 1). The incidence of SARS-CoV-2 infection (COVID-19) was $11.7 \%$ for the entire cohort, $8.6 \%$ for patients with cancer, $13.2 \%$ for patients with CVD, and 9.3\% for patients with both cancer and CVD.

\section{Patient Characteristics}

Baseline characteristics of patients with COVID-19 are shown in Table 1. Patients with COVID-19 and a history of cancer were older (aged 77 vs 57 years; $P<.001$ ) and more likely to be male ( $52 \%$ vs $41 \% ; P=.003$ ) compared with those without cancer. Furthermore, patients with cancer had a higher prevalence of cardiovascular risk factors and established CVD, CKD, and COPD, and were more likely to be on cardiovascular medications at baseline.
Patients with both cancer and CVD were older than those with either cancer (age 80 vs 72 years; $P<.001$ ) or CVD (age 80 vs 77 years; $P=.030$ ) alone. In addition to having a higher prevalence of cardiovascular risk factors and medications, patients with both cancer and CVD also had a higher prevalence of CKD compared with those with cancer alone ( $38 \%$ vs $14 \% ; P<.001)$. Other than older age and lower body mass index $\left(25.3\right.$ vs $27.8 \mathrm{~kg} / \mathrm{m}^{2}$; $P=.006$ ), there were no significant differences between patients with both cancer and CVD and those with CVD alone.

\section{Cancer Characteristics}

Cancer characteristics of patients with COVID-19 and cancer $(n=195)$ are shown in Table 2 . Breast cancer was the most prevalent malignancy $(n=37 ; 19 \%)$, followed by prostate cancer $(n=34 ; 17.4 \%)$. A total of 25 patients $(12.8 \%)$ had metastatic disease, and 27 (13.8\%) had a history of $>1$ cancer. A total of 48 patients $(24.6 \%)$ had active cancer or recent antineoplastic therapy, either at the time of SARS-CoV-2 infection or within the previous 6 months. For patients without

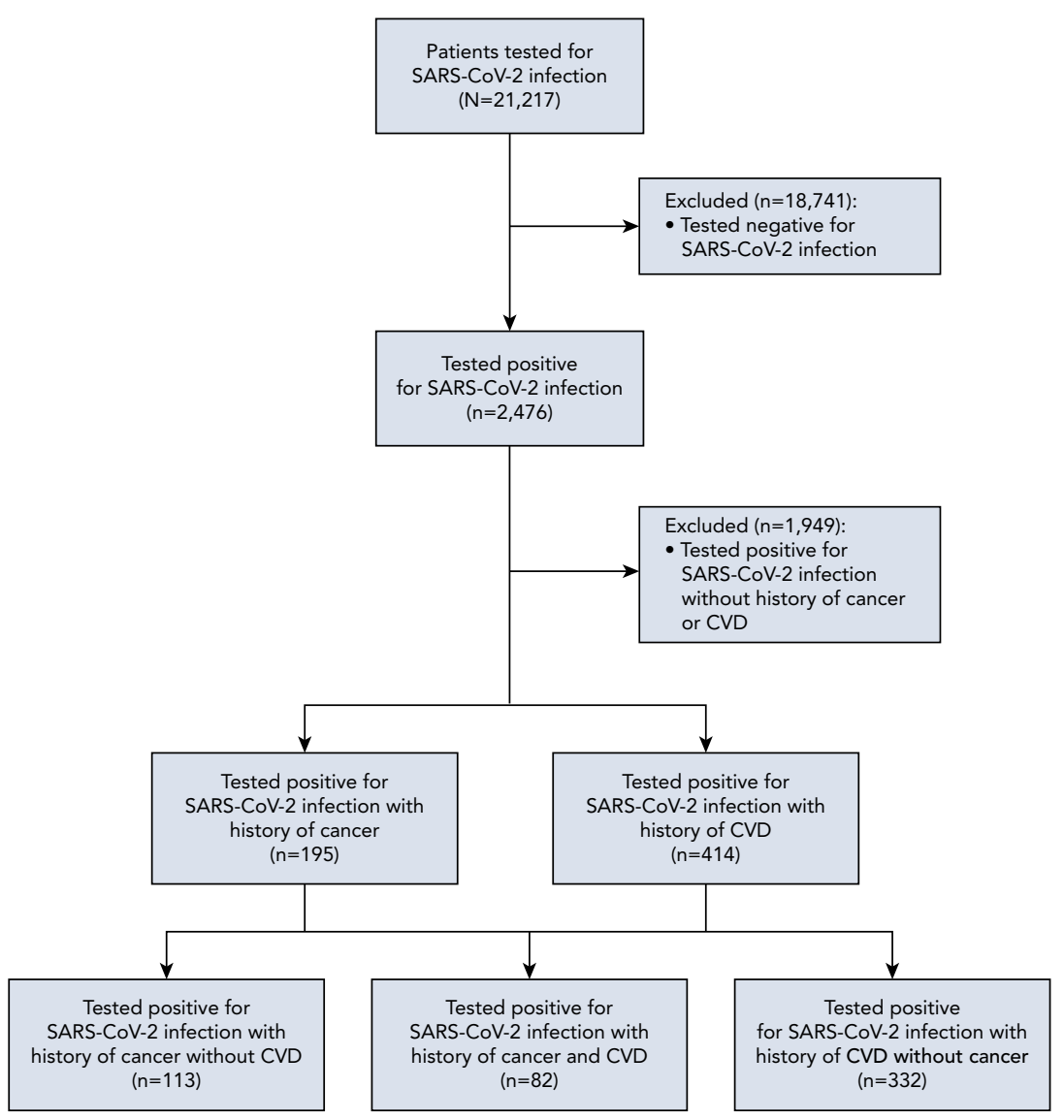

Figure 1. Proportion of patients who tested positive for SARS-CoV-2 stratified by a history of cancer or CVD, and subsequently by the presence of comorbid cancer and CVD.

Abbreviation: CVD, cardiovascular disease. 
Q

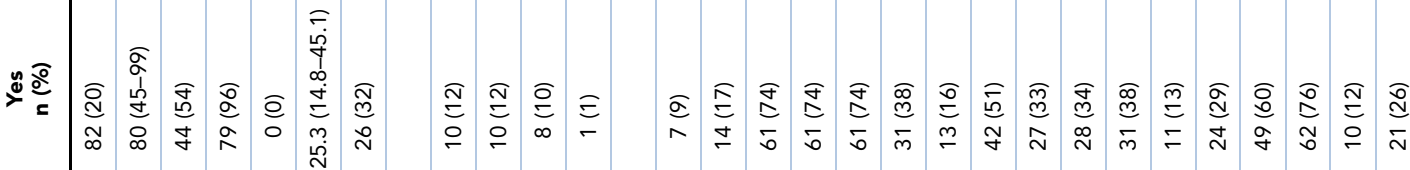

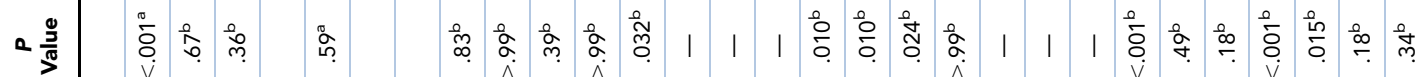

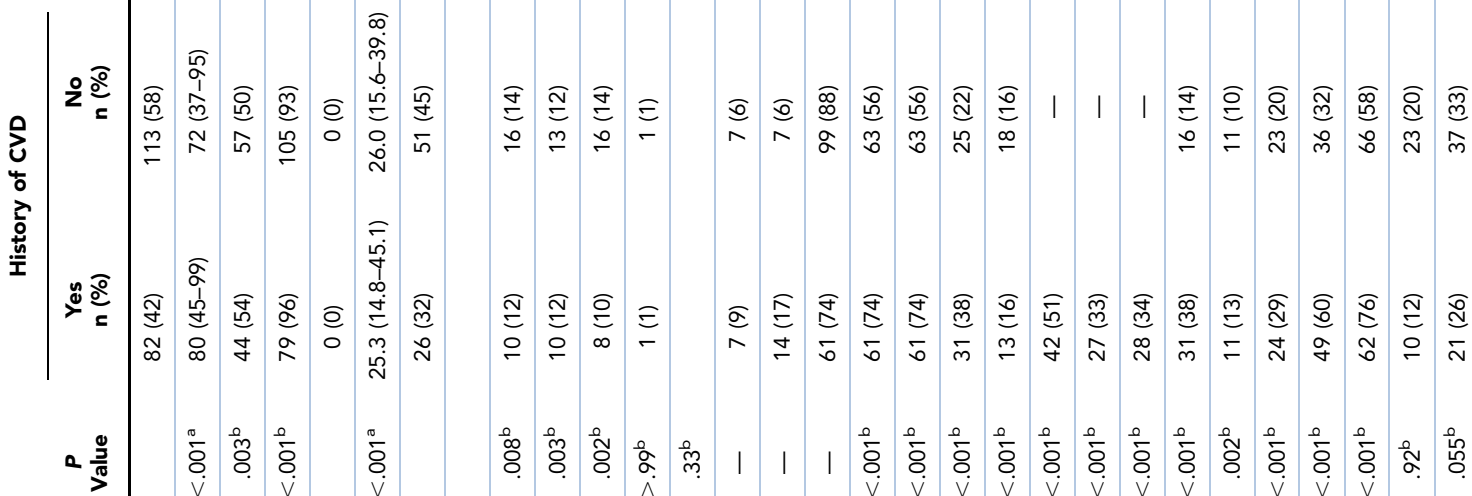

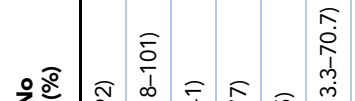

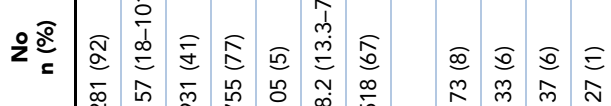

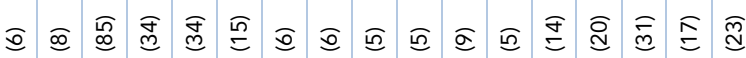

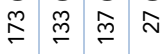

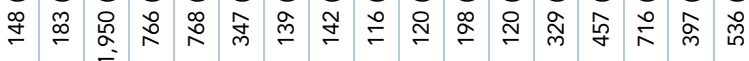

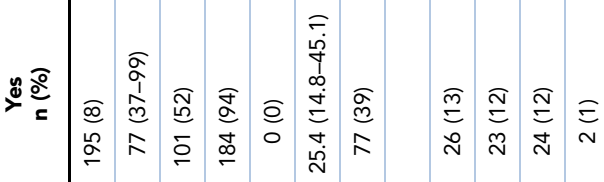

E) $\frac{0}{\square}$

\section{है}




\section{Table 2. Cancer Characteristics}

\begin{tabular}{|c|c|}
\hline Characteristic & n (\%) \\
\hline Total, N & $195(100)$ \\
\hline \multicolumn{2}{|l|}{ Cancer type } \\
\hline Breast & $37(19.0)$ \\
\hline Lung & $22(11.3)$ \\
\hline Kidney/Bladder & $19(9.7)$ \\
\hline Gastrointestinal & $17(8.7)$ \\
\hline Hematologic & $22(11.3)$ \\
\hline Prostate & $34(17.4)$ \\
\hline Skin & $16(8.2)$ \\
\hline Gynecologic & $9(4.6)$ \\
\hline Thyroid & $7(3.6)$ \\
\hline Testicular & $4(2.1)$ \\
\hline Head and neck & $6(3.1)$ \\
\hline Central nervous system & $2(1.0)$ \\
\hline Metastatic disease & $25(12.8)$ \\
\hline History of multiple cancers & $27(13.8)$ \\
\hline Cancer in remission at COVID-19 infection & $147(75.4)$ \\
\hline Ongoing antineoplastic therapy & $37(19.0)$ \\
\hline Median time from last therapy (range), mo & $65(7-508)$ \\
\hline Active or recent antineoplastic therapy ${ }^{a}$ & $48(24.6)$ \\
\hline \multicolumn{2}{|l|}{ Type of antineoplastic therapy } \\
\hline Anthracycline & $8(4.1)$ \\
\hline Anti-HER2 therapy & $6(3.1)$ \\
\hline Hormonal therapy & $27(13.8)$ \\
\hline Immune checkpoint inhibitor & $2(1.0)$ \\
\hline Tyrosine kinase inhibitor & $5(2.6)$ \\
\hline $5-\mathrm{FU}$ & $5(2.6)$ \\
\hline Cisplatin/Carboplatin/Oxaliplatin & $12(6.2)$ \\
\hline Rituximab & $10(5.1)$ \\
\hline Cyclophosphamide & $9(4.6)$ \\
\hline Paclitaxel & $9(4.6)$ \\
\hline Others $^{a}$ & $18(9.2)^{b}$ \\
\hline History of radiation therapy & $58(29.7)$ \\
\hline
\end{tabular}

ancludes bortezomib, daratumumab, pomalidomide, cetuximab, vincristine, etoposide, mitomycin, bendamustine, gemcitabine, pemetrexed, chlorambucil, cladribine, and taxotere.

bWithin 6 months before COVID-19 infection.

active/recent cancer, median time from antineoplastic therapy was 65 months (range, 7-508 months). Of these 195 patients, 27 (13.8\%) were exposed to hormonal therapy (for breast or prostate cancer), 8 (4.1\%) to anthracyclines, and 6 (3.1\%) to HER2-targeted agents, and $58(29.7 \%)$ had received regional radiation treatment. Few patients had received immune checkpoint inhibitors $(\mathrm{n}=2 ; 1.0 \%)$ or tyrosine kinase inhibitors $(\mathrm{n}=5 ; 2.6 \%)$.
Inflammatory Biomarkers

Data on inflammatory biomarkers were available for approximately one-third of patients with COVID-19, and showed that median values of peak D-dimer, ferritin, C-reactive protein, and IL-6 levels were similar in those with and without cancer (supplemental eTable 1).

\section{Medical Management of COVID-19}

Patients with COVID-19 and cancer received hydroxychloroquine ( \pm azithromycin) and steroids more often than those without cancer (Table 3). Remdesivir $(n=8$; $<1 \%)$ and tocilizumab $(n=38 ; 2 \%)$ were used infrequently in this cohort. Although tocilizumab use did not differ between patients with and without cancer, those with both cancer and CVD received tocilizumab more frequently than those with cancer alone ( $5 \%$ vs $0 \%$ ). Medical therapy did not differ significantly between those with both cancer and CVD and those with CVD alone (Table 3).

\section{COVID-19-Associated Severe Disease in Patients With Cancer}

In the entire cohort of patients with COVID-19, 273 (11\%) developed severe disease over a median of 22 days (range, 20-29 days). Multivariable analysis identified a history of cancer as an independent predictor of severe disease (Figure 2). Other significant predictors included advanced age, male sex, smoking history, diabetes, and use of $\beta$-blockers. After controlling for other factors, CVD was not an independent predictor of COVID-19associated severe disease in our cohort.

Cox regression analysis showed that patients with COVID-19 and cancer were at higher risk of developing severe disease (HR, 2.02; 95\% CI, 1.53-2.68; $P<.001)$ and had a shorter median time to severe disease (11 [range, $10-30$ ] vs 23 [range, 21-30] days; $P<.001$ ) than patients without cancer (Figure 3A). Even after exclusion of patients with active/recent cancer, those with cancer had a higher incidence of severe disease compared with those without cancer $(24.4 \%$ vs $11 \% ; P<.05)$. Univariate analyses identified active/recent cancer (odds ratio, 3.6; 95\% CI, 1.71-7.58; $P<.001$ ) and lung cancer (odds ratio, 3.30; 95\% CI, 1.29-8.42; $P<.013$ ) as risk factors for severe disease. However, patients with active/recent cancer had a similar mortality as those with a remote history of cancer (25\% vs $24.4 \% ; P=1.00$ ). Hematologic malignancies, metastatic cancer, exposure to radiation therapy, and exposure to cardiotoxic anticancer therapies were not associated with severe disease (supplemental eTable 2).

\section{COVID-19-Associated Severe Disease in}

\section{Patients With Cancer and Comorbid CVD}

Comorbid CVD was present in 82 patients $(42 \%)$ with a history of cancer. Those with both cancer and CVD were at higher risk of developing severe disease than those 
Table 3. In-Hospital Treatment of Patients With COVID-19

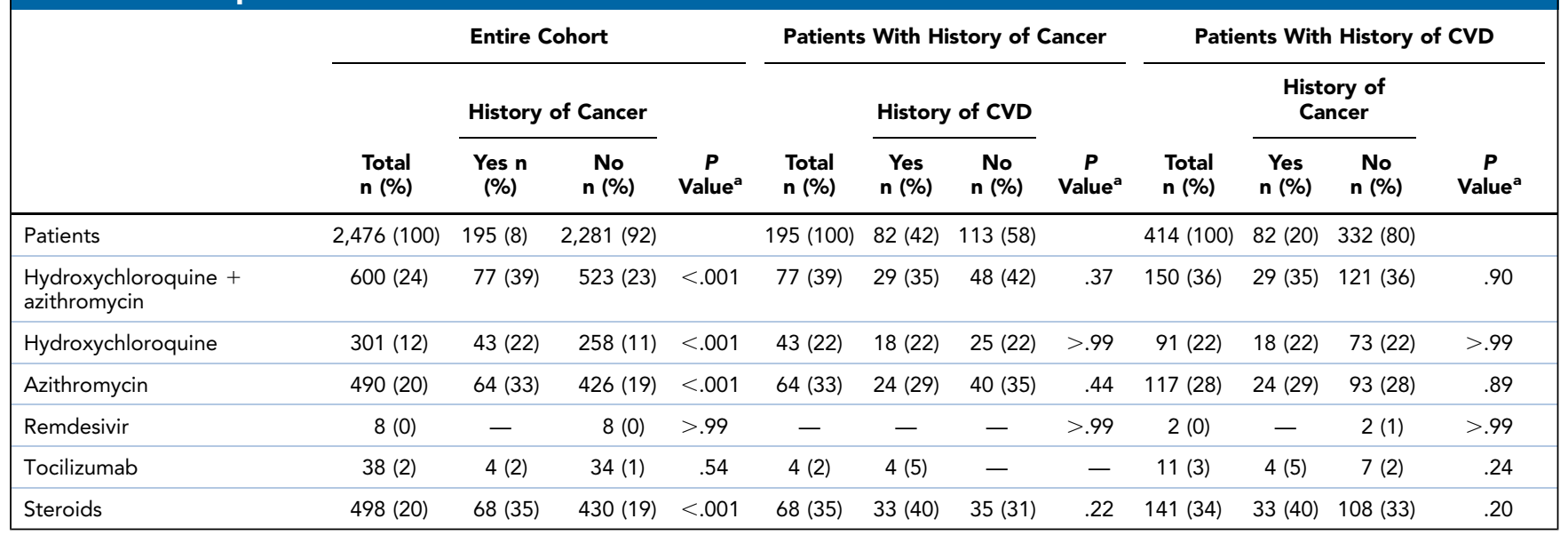

Abbreviation: CVD, cardiovascular disease.

aFisher exact test.

with cancer (HR, 1.86; 95\% CI, 1.11-3.10; $P=.018$; Figure 3B) or CVD alone (HR, 1.79; 95\% CI, 1.21-2.66; $P=.004$; Figure 3C). Furthermore, patients with cancer, CVD, or both were at higher risk of developing severe disease than those without cancer or CVD (Figure 3D).

\section{COVID-19-Associated Adverse Outcomes in \\ Patients With Cancer}

Adverse outcomes included as secondary endpoints are presented in Table 4. Patients with COVID-19 and cancer were hospitalized more frequently ( $65 \%$ vs $40 \% ; P<.001)$. Although the need for mechanical ventilation and supplemental oxygen did not differ between those with and without cancer, shock ( $8 \%$ vs $4 \% ; P=.011$ ), mortality ( $25 \%$ vs $8 \%$; $P<.001)$, arrhythmia ( $16 \%$ vs $5 \% ; P<.001)$, venous thromboembolism (10\% vs 3\%; $P<.001)$, and encephalopathy ( $13 \%$ vs $8 \% ; P=.019$ ) were significantly higher in patients with cancer than in those without cancer. Patients with cancer also had significantly longer median lengths of stay compared with those without cancer (5 vs 3 days; $P<.001$ ). Due to missing data, we did not detect a difference in the frequency of abnormal troponin levels between patients with and without cancer.

\section{COVID-19-Associated Adverse Outcomes in}

\section{Patients With Cancer and Comorbid CVD}

Patients with COVID-19 and both cancer and CVD died more frequently than those with cancer $(35 \%$ vs $17 \%$; $P=.004$ ) or CVD (35\% vs $21 \%$; $P=.009$ ) alone. Patients with cancer and CVD also had a higher frequency of arrhythmias (24\% vs $10 \%$; $P=.009$ ) and encephalopathy ( $21 \%$ vs $7 \%$; $P=.008$ ) compared with those with cancer alone. There were no significant differences in other adverse effects between patients with both cancer and CVD and those with either diagnosis alone (Table 4).

\section{Discussion}

Cancer and CVD have been identified as risk factors for adverse outcomes in patients with COVID-19. In this analysis, patients with a history of cancer were at higher risk of requiring hospitalization and experiencing adverse outcomes than those without cancer, especially if they had comorbid CVD. This is the first study to evaluate the impact of a dual diagnosis of cancer and CVD on COVID-19 outcomes.

Prior studies from China found that among patients hospitalized with COVID-19, the proportion with a history of cancer exceeded the incidence of cancer in the general population..$^{5,9}$ This raised the concern that patients with a history of cancer might be at higher risk of acquiring COVID-19. In our study, the incidence of infection in patients with cancer did not exceed the incidence in the overall cohort of patients with COVID19 , suggesting that patients with cancer may not be at increased risk of acquiring COVID-19. Similarly, the incidence of infection in patients with both cancer and CVD was also not higher than that observed in the overall cohort.

This study is the first to evaluate relative hospitalization rates in patients with COVID-19 with a history of cancer. Our results showed that patients with cancer required hospitalization more frequently than those without cancer. Interestingly, the rate of hospitalization in patients with both cancer and CVD did not differ significantly from the rates in patients with either cancer or CVD alone, perhaps because of the small number of patients.

Prior studies have shown that patients with COVID19 and a history of cancer have significantly increased mortality compared with noncancer control patients. A study from China compared outcomes between 109 


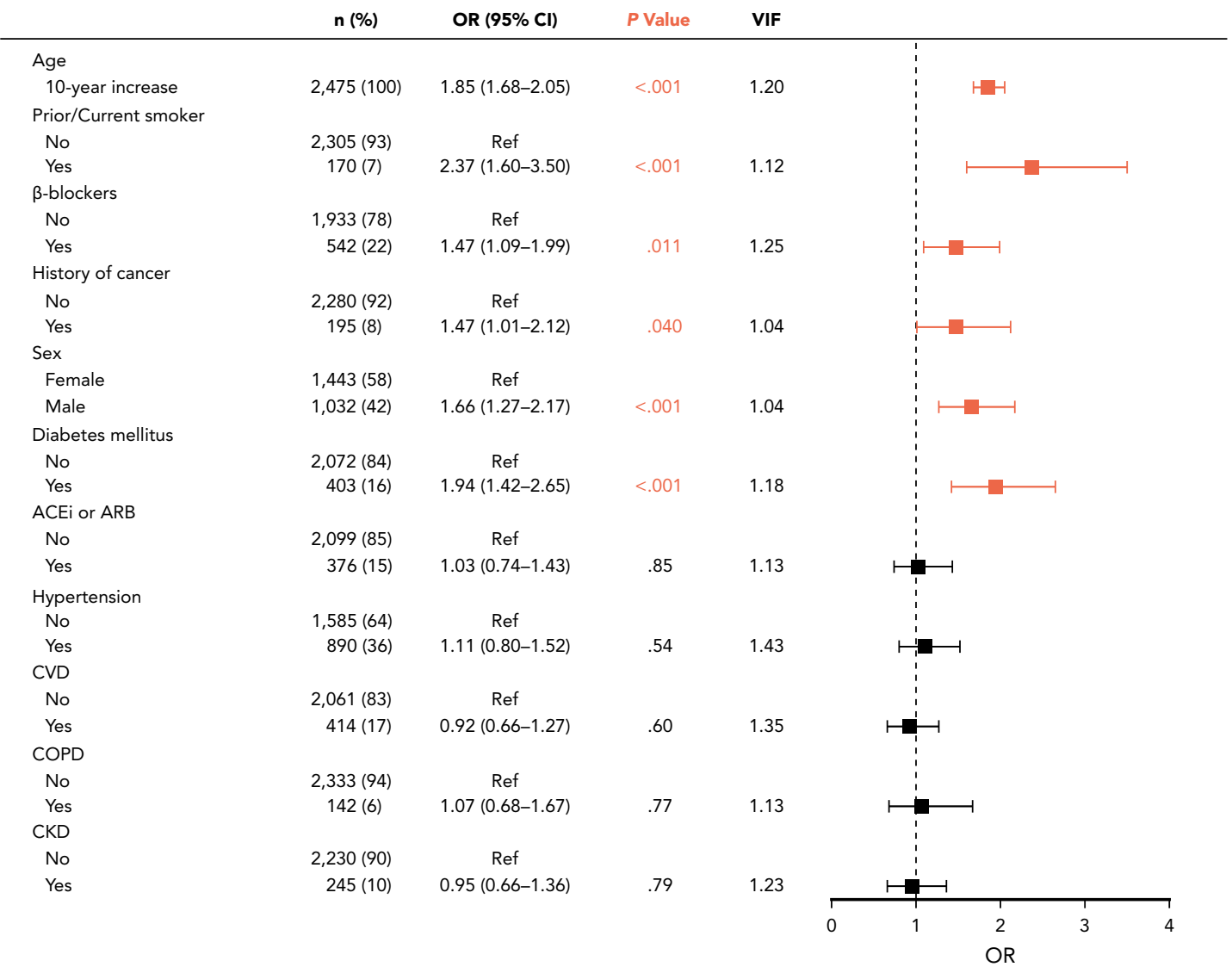

Figure 2. Predictors of severe disease in all patients with COVID-19. Use of ACEi or ARB, hypertension, CVD, COPD, and CKD were not associated with the development of severe disease. VIFs were computed for the final model to evaluate multicollinearity. Abbreviations: ACEi, angiotensin-converting enzyme inhibitors; ARB, angiotensin receptor blockers; CKD, chronic kidney disease; COPD, chronic obstructive pulmonary disease; CVD, cardiovascular disease; OR, odds ratio; VIF, variance inflation factor.

patients with cancer and 327 propensity-matched noncancer control patients and found a 3 -fold higher risk of mortality in those with cancer. ${ }^{10}$ Similarly, another study from China compared 105 patients with cancer with 536 age-matched, noncancer control patients and found that those with cancer had a 2- to 3-fold higher risk of serious adverse effects, including admission to the ICU, need for mechanical ventilation, and death. ${ }^{11}$ In a study from New York City, the case fatality rate among 218 patients with COVID-19 and cancer was 2 -fold higher than that observed in 1,090 noncancer control patients. ${ }^{12}$ Results of our study concur with these prior studies and show that patients with COVID19 and a history of cancer have a 3-fold higher risk of death. Furthermore, multivariable logistic regression analysis confirmed that among patients with COVID19 , a history of cancer was independently associated with severe disease, defined as a composite of the need for mechanical ventilation, shock, or death. In addition, our study showed that patients with COVID-19 and cancer are also at an increased risk of developing other complications, including arrhythmias, venous thromboembolism, and encephalopathy.

Two large registry-based studies of patients with cancer and COVID-19 from the United Kingdom ${ }^{13}$ and the United States ${ }^{4}$ evaluated cancer characteristics that predict mortality. Kuderer et $\mathrm{al}^{4}$ found a higher risk of mortality in patients receiving active/recent chemotherapy. Unlike other studies, these registries did not identify lung cancer, ${ }^{12}$ hematologic malignancies, ${ }^{11,12}$ or metastatic disease ${ }^{11}$ as predictors of mortality. ${ }^{4,13}$ Our study did not have adequate events to evaluate predictors of mortality in patients with cancer using multivariable regression analysis. However, in univariate analyses, a history of lung cancer and active/recently treated cancer were associated with a higher incidence of severe COVID-19-related disease. Notably, in this analysis, even patients with cancer in remission were at increased risk of severe disease compared with those without cancer.

Cardiovascular risk factors and CVD have been associated with adverse outcomes in patients with COVID-19. 
A

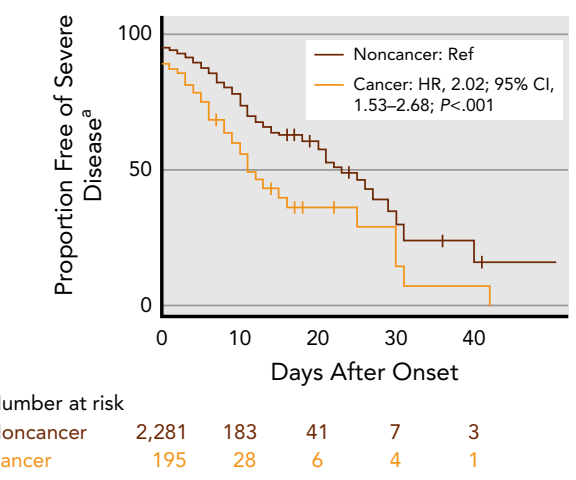

C

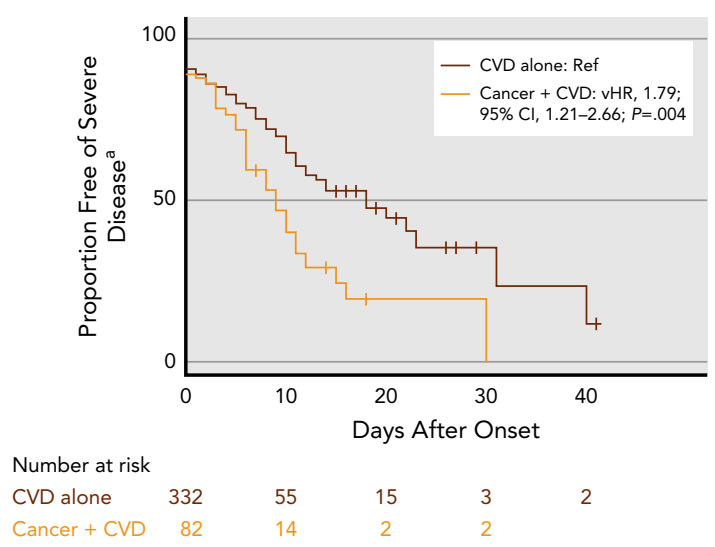

B

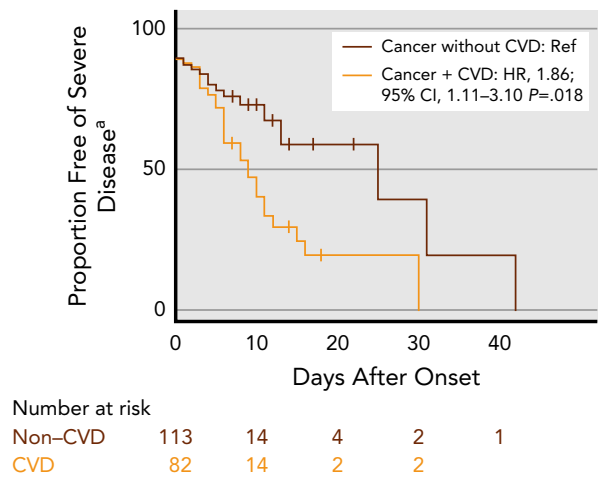

D

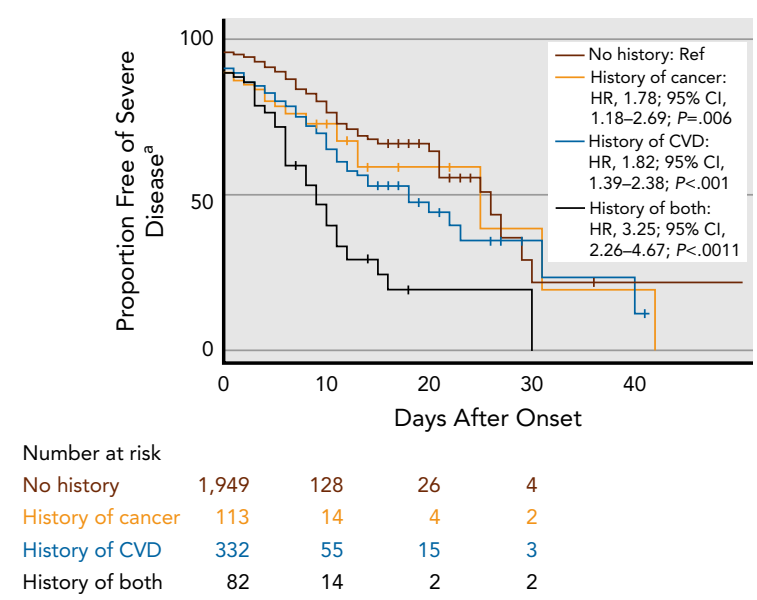

Figure 3. Impact of cancer and CVD on the development of severe disease. Kaplan-Meier survival curves for severe-disease-free survival in patients who were SARS-CoV-2-positive (A) stratified by history of cancer; (B) with cancer, stratified by CVD status; (C) with CVD, stratified by presence or absence of cancer; and (D) with no history of either CVD or cancer, history of cancer without CVD, history of CVD without cancer, and history of both cancer and CVD.

Abbreviations: CVD, cardiovascular disease; $\mathrm{HR}$, hazard ratio.

aDefined as the composite of the need for mechanical ventilation, shock, or death.

In a study of 187 patients with COVID-19 from China, one-third had underlying CVD and a 2-fold higher mortality than those without CVD. ${ }^{14}$ Similarly, in another study from China comparing 137 survivors of COVID-19 with 54 nonsurvivors, underlying hypertension, diabetes, and coronary artery disease were significantly associated with mortality. ${ }^{7}$ A study from Italy found that more than half of 99 patients hospitalized with COVID-19 had underlying CVD, with a $36 \%$ mortality rate compared with $15 \%$ in those without CVD. ${ }^{15}$ In the current study, multivariable analysis did not identify CVD as an independent predictor of severe disease. This finding may have resulted from collinearity with other significant predictors, such as advanced age, history of diabetes mellitus, and use of $\beta$-blockers. In addition, $\beta$-blockers may have contributed to adverse outcomes via their bronchospastic or negative inotropic effects. However, in a large case-control study from Italy, $\beta$-blocker use was not associated with critical or fatal disease. ${ }^{16}$

Cancer and CVD are the 2 largest causes of death in the United States and often exist together. ${ }^{17,18}$ In fact, almost half of the patients with cancer in our study had comorbid CVD. We hypothesized that patients with a dual diagnosis of cancer and CVD would have worse outcomes than those with cancer or CVD alone. In the UK registry of 800 patients with active cancer and symptomatic COVID-19, underlying CVD was a significant univariate predictor of mortality. ${ }^{13}$ Our results extend these findings and show that although patients with either cancer or CVD are at increased risk, those with a dual diagnosis of cancer and CVD are at even higher risk of COVID-19-associated severe disease. These findings have important public health implications with regard to social distancing and in-person medical encounters for 


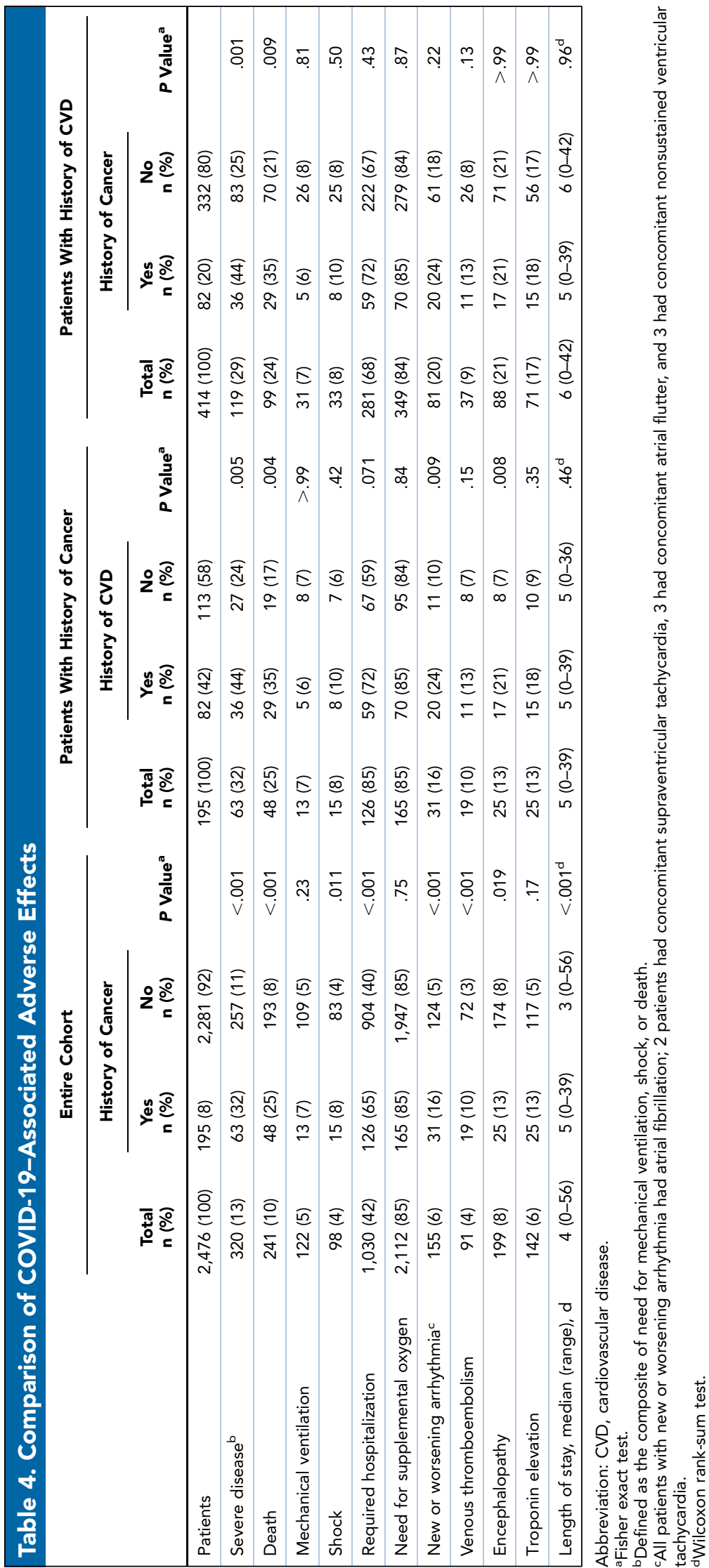


patients with cancer and comorbid CVD. Patients with cancer with underlying CVD are considered high-risk for cardiotoxicity and are recommended to have periodic screening during treatment with potentially cardiotoxic cancer therapies. ${ }^{19}$ Given their heightened risk for adverse outcomes with COVID-19, the need and frequency of such surveillance should be reevaluated on a case-bycase basis. Ongoing utilization of telehealth and home health services for such patients in areas of high viral transmission should be strongly considered.

This study had some limitations. Because universal testing has not been instituted in the United States, we may have underestimated or overestimated the incidence of COVID-19 in patients with cancer due to referral bias. Data regarding race, body mass index, and inflammatory markers were missing in many patients (Table 1 and supplemental eTable 1), thus limiting assessment of their impact on outcomes. Similarly, assessment of troponin level elevation as an adverse outcome was also limited due to missing values. In addition, the number of patients with cancer was too small to allow multivariable assessment of predictors of adverse outcomes in this population. Furthermore, although chart review suggested that the etiology of severe disease for all patients was COVID-19-related, it is certainly possible that patients with underlying comorbid conditions experienced worsening of their preexisting disease, which may have contributed to the severity of their illness. Finally, a very small proportion of our cohort had active/recently treated cancer, preventing us from assessing the impact of specific cancer therapies on the risks associated with COVID-19.

\section{Conclusions}

The findings of this study suggest that patients with a history of cancer, particularly those with comorbid CVD, are at a significantly higher risk of COVID-19-associated adverse outcomes and need to be counseled accordingly. With the advent of effective therapies and emerging vaccines, these patients may need to be prioritized as a high-risk group for these therapies.

Submitted July 1, 2020; accepted for publication September 23, 2020.

Author contributions: Study design: Ganatra, Dani, Baron, Nohria. Data collection: Ganatra. Data collection and organization: R. Patel, Parikh, Bang, Shreyder, Brar, Singh, Kumar. Electronic medical record informatics and data collection: Rieger-Christ. Data analysis: Dani, Redd, Kazi, Guha, Hayek. Expertise about cancer and cardiovascular disease: Asnani, Barac, Gunturu, Zarwan, Mosenthal, Yunus, J.M. Patel, Patten, Venesy, Shah, Resnic. Data interpretation: Ganatra, Dani, Baron, Nohria. Manuscript preparation, with input from all authors: Ganatra, Dani, Baron, Nohria.

Disclosures: Dr. Nohria has disclosed that she has received grant/research support from Amgen, is a scientific advisor for Takeda Oncology, and has received consulting fees from AstraZeneca. The authors have disclosed that they have not received any financial consideration from any person or organization to support the preparation, analysis, results, or discussion of this article.

Correspondence: Sarju Ganatra, MD, Division of Cardiovascular Medicine, Lahey Hospital and Medical Center, 41 Mall Road, Burlington, MA 01805. Email: Sarju.ganatra@lahey.org

\section{References}

1. Report of the WHO-China Joint Mission on Coronavirus Disease 2019 (COVID-19). Accessed September 30, 2020. Available at: https://www. who.int/docs/default-source/coronaviruse/who-china-joint-mission-oncovid-19-final-report.pdf

2. Ganatra S, Hammond SP, Nohria A. The novel coronavirus disease (COVID-19) threat for patients with cardiovascular disease and cancer. JACC CardioOncol 2020;2:350-355.

3. Ganatra S, Dani SS, Shah S, et al. Management of cardiovascular disease during coronavirus disease (COVID-19) pandemic. Trends Cardiovasc Med 2020;30:315-325.

4. Kuderer NM, Choueiri TK, Shah DP, et al. Clinical impact of COVID-19 on patients with cancer (CCC19): a cohort study. Lancet 2020;395: 1907-1918.

5. Liang W, Guan W, Chen R, et al. Cancer patients in SARS-CoV-2 infection: a nationwide analysis in China. Lancet Oncol 2020;21: 335-337.

6. Guan WJ, Ni ZY, Hu Y, et al. Clinical characteristics of coronavirus disease 2019 in China. N Engl J Med 2020;382:1708-1720.

7. Zhou F, Yu T, Du R, et al. Clinical course and risk factors for mortality of adult inpatients with COVID-19 in Wuhan, China: a retrospective cohort study. Lancet 2020;395:1054-1062.

8. Addison D, Campbell CM, Guha A, et al. Cardio-oncology in era of the COVID-19 pandemic and beyond [published online July 27, 2020]. J Am Heart Assoc, doi: 10.1161/JAHA.120.017787

9. Yu J, Ouyang W, Chua MLK, et al. SARS-CoV-2 transmission in patients with cancer at a tertiary care hospital in Wuhan, China. JAMA Oncol 2020; $6: 1108-1110$.
10. Meng Y, Lu W, Guo E, et al. Cancer history is an independent risk factor for mortality in hospitalized COVID-19 patients: a propensity score-matched analysis. J Hematol Oncol 2020;13:75.

11. Dai M, Liu D, Liu M, et al. Patients with cancer appear more vulnerable to SARS-CoV-2: a multicenter study during the COVID-19 outbreak. Cancer Discov 2020;10:783-791.

12. Mehta V, Goel S, Kabarriti R, et al. Case fatality rate of cancer patients with COVID-19 in a New York hospital system. Cancer Discov 2020;10:935-941.

13. Lee LYW, Cazier JB, Angelis V, et al. COVID-19 mortality in patients with cancer on chemotherapy or other anticancer treatments: a prospective cohort study. Lancet 2020;395:1919-1926.

14. Guo T, Fan Y, Chen M, et al. Cardiovascular implications of fatal outcomes of patients with coronavirus disease 2019 (COVID-19). JAMA Cardiol 2020;5:811-818

15. Inciardi RM, Adamo M, Lupi L, et al. Characteristics and outcomes of patients hospitalized for COVID-19 and cardiac disease in Northern Italy. Eur Heart J 2020;41:1821-1829

16. Mancia $G$, Rea F, Ludergnani $M$, et al. Renin-angiotensin-aldosterone system blockers and the risk of COVID-19. N Engl J Med 2020;382: 2431-2440.

17. DeSantis $C E$, Lin $C C$, Mariotto $A B$, et al. Cancer treatment and survivorship statistics, 2014. CA Cancer J Clin 2014;64:252-271.

18. Ganatra S, Hayek SS. Cardio-oncology for GenNext: a missing piece of the training puzzle. J Am Coll Cardiol 2018;71:2977-2981.

19. Curigliano G, Lenihan $D$, Fradley $M$, et al. Management of cardiac disease in cancer patients throughout oncological treatment: ESMO consensus recommendations. Ann Oncol 2020;31:171-190. 
Supplemental online content for:

\section{Outcomes of COVID-19 in Patients With a History of Cancer and Comorbid Cardiovascular Disease}

Sarju Ganatra, MD; Sourbha S. Dani, MD; Robert Redd, MS; Kimberly Rieger-Christ, PhD; Rushin Patel, MD; Rohan Parikh, MD; Aarti Asnani, MD; Vigyan Bang, MD; Katherine Shreyder, MD; Simarjeet S. Brar, MD; Amitoj Singh, MD; Dhruv S. Kazi, MD; Avirup Guha, MD; Salim S. Hayek, MD; Ana Barac, MD; Krishna S. Gunturu, MD; Corrine Zarwan, MD; Anne C. Mosenthal, MD; Shakeeb A. Yunus, MD; Amudha Kumar, MD; Jaymin M. Patel, MD; Richard D. Patten, MD; David M. Venesy, MD; Sachin P. Shah, MD; Frederic S. Resnic, MD;

Anju Nohria, MD; and Suzanne J. Baron, MD

J Natl Compr Canc Netw, doi: 10.6004/jnccn.2020.7658

eTable 1: Biomarkers

eTable 2: Presence of Severe Disease Based on Cancer Characteristics eAppendix 1: Definitions of Individual Endpoints 
eTable 1. Biomarkers

Total Cohort

History of Cancer

$\begin{array}{ccc}\text { Yes } & \text { No } & P \\ \text { n (\%) } & \text { n (\%) } & \text { Value }\end{array}$

\begin{tabular}{|c|c|c|c|c|c|c|c|}
\hline & n (\%) & $n(\%)$ & n (\%) & value & n (\%) & n (\%) & value \\
\hline Patients & $2,476(100)$ & $195(8)$ & $2,281(92)$ & & $82(42)$ & $113(58)$ & \\
\hline $\begin{array}{l}\text { Nadir WBC count, } \\
\text { median (range), K/ } \mu \mathrm{L}\end{array}$ & $0.42(0.01-1.80)$ & $0.33(0.01-1.25)$ & $0.43(0.01-1.80)$ & $.76^{\mathrm{a}}$ & $0.26(0.01-1.09)$ & $0.47(0.01-1.25)$ & $.2^{\mathrm{a}}$ \\
\hline Missing & $1,689(68)$ & $90(46)$ & $1,599(70)$ & & $32(39)$ & $58(51)$ & \\
\hline Lymphopenia, n (\%) & $5(0)$ & $1(1)$ & $4(0)$ & $.51^{b}$ & - & $1(1)$ & $>.99^{b}$ \\
\hline Missing & $1,583(64)$ & $75(38)$ & $1,508(66)$ & & $24(29)$ & $51(45)$ & \\
\hline $\begin{array}{l}\text { Peak D-dimer, median } \\
\text { (range), } \mu \mathrm{g} / \mathrm{mL}\end{array}$ & $885(200-20,000)$ & $865(200-18,630)$ & $898(200-20,000)$ & $.92^{\mathrm{a}}$ & $840(200-18,630)$ & $890(200-3,908)$ & $.76^{\mathrm{a}}$ \\
\hline Missing & $1,960(79)$ & $125(64)$ & $1,835(80)$ & & $49(60)$ & $76(67)$ & \\
\hline $\begin{array}{l}\text { Peak ferritin, median } \\
\text { (range), } \mathrm{ng} / \mathrm{mL}\end{array}$ & $730(16-30,506)$ & $786(24-11,202)$ & $728(16-30,506)$ & $.54^{b}$ & $795(76-11,202)$ & $786(24-4,240)$ & $.50^{a}$ \\
\hline Missing & $1,885(76)$ & $111(57)$ & $1,774(78)$ & & 40 (49) & $71(63)$ & \\
\hline $\begin{array}{l}\text { Peak CRP, median } \\
\text { (range), } \mathrm{mg} / \mathrm{mL}\end{array}$ & $116.3(0.8-620.9)$ & $118.6(3.6-410.5)$ & $116.0(0.8-620.9)$ & $.90^{\mathrm{a}}$ & $120.6(20.6-410.5)$ & $112.0(3.6-408.1)$ & $.63^{a}$ \\
\hline Missing & $1,799(73)$ & $96(49)$ & $1,703(75)$ & & $34(41)$ & $62(55)$ & \\
\hline $\begin{array}{l}\text { Peak troponin, median } \\
\text { (range), ng/mL }\end{array}$ & $0.03(0.01-230.19)$ & $0.04(0.01-230.19)$ & $0.02(0.01-34.28)$ & $<.001^{a}$ & $0.04(0.01-230.19)$ & $0.03(0.01-1.41)$ & $.21^{a}$ \\
\hline Missing & $1,816(73)$ & $103(53)$ & $1,713(75)$ & & $36(44)$ & 67 (59) & \\
\hline $\begin{array}{l}\text { Peak IL-6, median } \\
\text { (range), } \mathrm{pg} / \mathrm{mL}\end{array}$ & $19.5(3.2-577.0)$ & $14.5(5.0-445.0)$ & $19.5(3.2-577.0)$ & $.75^{a}$ & $445(445-445)$ & $6(5-30)$ & $.29^{b}$ \\
\hline Missing & $2,388(96)$ & $187(96)$ & $2,201(96)$ & & $80(98)$ & $107(95)$ & \\
\hline $\begin{array}{l}\text { Peak creatinine, median } \\
\text { (range), } \mathrm{mg} / \mathrm{dL}\end{array}$ & $1.2(0.5-20.5)$ & $1.3(0.6-9.8)$ & $1.1(0.5-20.5)$ & $.058^{a}$ & $1.6(0.7-9.8)$ & $1.2(0.6-5.6)$ & $.011^{a}$ \\
\hline Missing & $1,571(63)$ & $74(38)$ & $1,497(66)$ & & $24(29)$ & $50(44)$ & \\
\hline
\end{tabular}

Abbreviations: CRP, C-reactive protein; CVD, cardiovascular disease.

aWilcoxon rank-sum test.

bFisher exact test. 


\section{eTable 2. Presence of Severe Disease Based on Cancer Characteristics}

Severe Disease $^{a}$

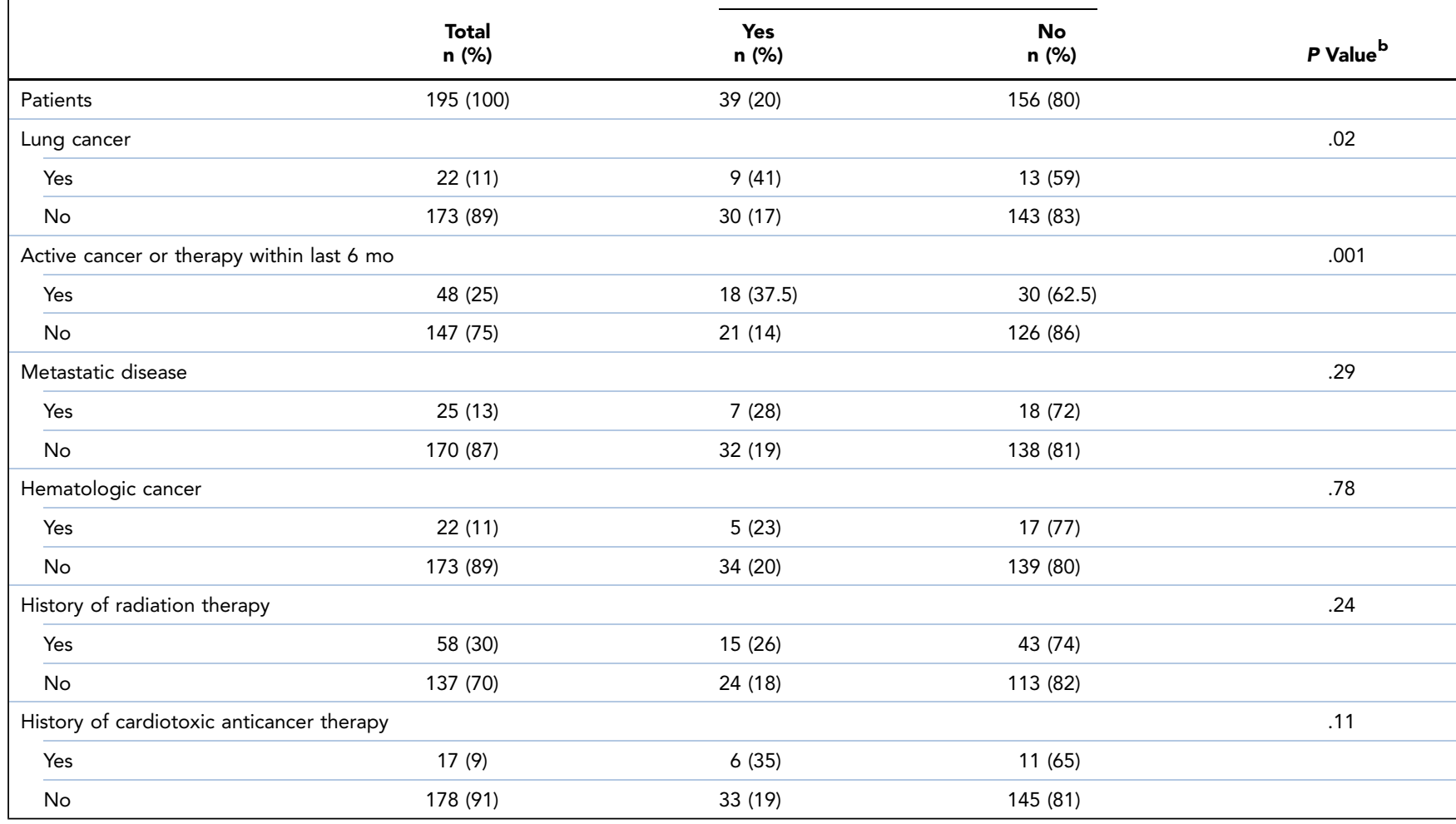

aSevere disease is defined as the composite of need for mechanical ventilation, shock, or death. Cardiotoxic anticancer therapies included anthracyclines, trastuzumab, and androgen deprivation therapy.

bFisher exact test. 


\section{eAppendix 1. Definitions of Individual Endpoints}

Shock was defined as vasopressor use $\geq 3$ hours to maintain organ perfusion. Cardiac troponin $\mathrm{I}>0.08 \mathrm{ng} / \mathrm{mL}$ was considered abnormal. Encephalopathy was defined as acute or subacute global, functional alteration of mental status due to systemic factors. Arrhythmia was defined as either new-onset arrhythmia (supraventricular tachycardia, atrial fibrillation, ventricular arrhythmia) requiring intervention or worsening of preexisting arrhythmia that required additional intervention. Chronic kidney disease was defined as kidney damage or glomerular filtration rate $<60 \mathrm{~mL} / \mathrm{min} / 1.73 \mathrm{~m}^{2}$ for $\geq 3$ months irrespective of cause, and/or the presence of albuminuria, defined as albumin-to-creatinine ratio $>30 \mathrm{mg} / \mathrm{g}$ in 2 of 3 spot urine specimens or history of kidney transplant. 